\title{
ABDUCTIVE INFERENCES IN STRATEGIC DESIGN DECISIONS
}

\author{
Kroll, Ehud (1); Koskela, Lauri (2) \\ 1: ORT Braude College; 2: University of Huddersfield
}

\begin{abstract}
The overall strategy of designing is addressed. The design decisions that have a major impact on the direction in which the process evolves are termed "strategic", and here we study them from the perspective of abduction. The aim is to clarify the role of abduction (in the sense of inference to the best explanation) in strategic decision making in design. Four cases are used for demonstration and discussion: functional decomposition in novel situations; the ordering of subfunctions in a function structure; the order of development of design tasks; and managing the design iterations. We focus on two specific design strategies: systematic design and parameter analysis, and show that strategic abductions often take place within the chosen strategy for the sake of efficiency of the process. Such abductions are often triggered by rules (like focusing first on the issue with greatest uncertainty in the total design task) that derive from Peirce's principle for economy of research. It is found that strategic abductions may have a decisive impact on the outcome of a design process. Two potential ways of improving design strategies and related strategic abductions are discussed.
\end{abstract}

Keywords: Abduction, Creativity, Design cognition, Design process

\section{Contact:}

Kroll, Ehud

ORT Braude College

Mechanical Engineering

Israel

kroll@braude.ac.il

Cite this article: Kroll, E., Koskela, L. (2019) 'Abductive Inferences in Strategic Design Decisions', in Proceedings of the 22nd International Conference on Engineering Design (ICED19), Delft, The Netherlands, 5-8 August 2019. DOI:10.1017/dsi.2019.111 


\section{INTRODUCTION}

In the second half of the 20th century, in discussions about scientific discoveries, an inference called abduction by the American philosopher Peirce started to be addressed. Peirce had seen abduction as a peculiar type of inference that generates explanatory hypotheses, and as the only mental operation producing novelty. This discussion continues to be lively in philosophy of science. Somewhat later, it was proposed that abduction is at the heart of design too (March, 1976). Likewise, in the domain of design, a discussion about the role of abduction has emerged (Roozenburg, 1993; Dorst, 2011; Kroll and Koskela, 2016; Koskela et al., 2018).

However, abduction has turned out to be a somewhat confusing topic, for two main reasons. First, design as a context for abduction is in many ways different from science (Koskela et al., 2018). Peirce viewed that in science, an abduction is triggered by an anomaly, a surprising observation. Through abduction, an explanatory hypothesis is then generated. As one of the most significant differences, it has been proposed that in design, correspondingly, abduction is triggered by a problem that a designer cannot solve through habitual or known means. Then, through abduction, a creative solution emerges. Also, in the design context, abduction is proposed to be seen as a property of an inference rather than an inference itself (Koskela et al., 2018).

Another problem is that the notion of abduction has evolved, and nowadays this term is used in two different, although overlapping senses (Douven, 2017), which are not always clearly distinguished. In addition to the original, Peircean understanding of abduction as generation of hypotheses (discussed above), the understanding of abduction in terms of justification of hypotheses has gained foothold. In the latter case, abduction is seen as an inference to the best explanation (IBE). In simple terms, the main difference between these two understandings is that a Peircean abduction creates a novel explanation, whereas an abduction in the IBE sense chooses the best explanation among alternatives. A medical diagnosis is a good example of the latter: based on often incomplete and vague information, a doctor infers the best explanation for symptoms and prescribes the corresponding remedies. In contrast, a doctor having a novel insight into the ways of contagion of a disease represents the Peircean abduction.

As novelty is much sought after in design, the majority of studies into design abduction have focused primarily on the Peircean abduction. There, designing consists of inferences from a desired resultmost often, functions to be satisfied - to an artifact, a form or structure, that is proposed for that purpose. In this paper, however, abduction in the IBE sense is focused on. Design processes contain reasoning steps and decisions that do not directly affect the evolving artifact, as they do not specify features of the solution. Rather, these steps are concerned with the unfolding of the design process. In other words, many design activities follow a plan, which is the designer's strategy for handling the design task. We call the decisions that shape the design effort strategic design decisions. We contend that such decisions are very often based on IBE abduction: the possible moves are usually very well known to the designer, so that as such, any decision does not bring novelty, but the distinguishing criterion is to interpret the situation in the best way and based on that, decide on the most suitable course of action.

Many design theories and models - for example, axiomatic design (Suh, 1990), function-behaviorstructure (Gero and Kannengiesser, 2004) and C-K theory (Hatchuel and Weil, 2009) - are descriptive in nature, so they do not specify a strategy for the designer to use. In contrast, prescriptive models include a sort of algorithm that the designer is supposed to follow. For example, systematic design methods, such as Pahl \& Beitz's (Pahl et al., 2007), advocate abstracting of the customer needs to form a main functional need statement, functional decomposition to create a function structure, morphological search for solutions for the subfunctions, and finally, combining the solutions to form a proposed conceptual design. Such approaches are sometimes called "rule-based design", as they assume that all the functionalities are known at the beginning of the process, can be decomposed, and that existing solutions can be found for the subfunctions. A sort of deductive logic assures that combinations of solutions to the subfunctions can satisfy the overall function. This strategy combines the principles of divide and conquer with breadth-first exploration. Systematic methods are considered more suitable for routine design tasks than innovative ones (Kroll, 2013).

Other strategies are also possible, for example, opportunistic approaches encourage the designer to start designing in an ad-hoc manner, and handle the design issues as they are encountered. This usually leads to an unstructured, depth-first process, that may be typical of some expert designers (Cross, 2006). 
Another strategy is the co-evolution of problem and solution (Maher, 2001), where instead of spending considerable time on studying the problem and formulating the specifications at the beginning, the designer quickly starts with synthesizing solutions while updating the requirements along the way. Cross (2006) points to the fact that expert designers tend to have a strategy that is more of a flexible mixture of the depth-first and breadth-first approaches.

Choosing what strategy to use is a sort of selective abductive inference where one strategy is picked from a repertoire of strategies. This may be done at the beginning of the design process - in which case the selected strategy is followed throughout the process-or the designer may switch between strategies at any point. In what follows we will show that design strategies contain many needs and opportunities for abductive inferences at the level we call strategic, that is, they are related to the execution of the strategy and not directly to the designed artifact.

Thus, the aim of this article is to clarify the role of abduction (in the IBE sense) in strategic decision making in design, using four cases for demonstration and discussion: (a) functional decomposition in novel situations, (b) the ordering of sub-functions in a function structure, (c) the order of development of design tasks, and (d) managing the design iterations. We focus on two specific design strategies: systematic design (Pahl et al., 2007) and parameter analysis (Kroll et al., 2001), and show that strategic abductions often take place within the chosen strategy for the sake of efficiency of the process, as explained next.

\section{DESIGN PROCESS EFFICIENCY}

Although not well-known, there is actually a theoretical guideline that can be applied to increase the economy of design processes, as presented in 1879 by Peirce (1967), who stated, in the context of research:

The utility of knowledge consists in its capability of being combined with other knowledge so as to enable us to calculate how we should act. If the knowledge is uncertain, we are obliged to do more than is really necessary, in order to cover this uncertainty. And, thus, the utility of any increase of knowledge is measured by the amount of wasted effort it saves us, multiplied by the specific cost of that species of effort.

We contend that this principle directly applies also to design as there is uncertain or missing knowledge, and this uncertainty can be reduced or minimized through design activities and tests but also through research. Thus, the objective is to reduce the cost of wasted efforts. Especially, there are two types of wasted efforts that need consideration in this context:

1. The wasted cost of design efforts if the intended design turns out to be impossible. If a novel artifact is targeted, and there is uncertainty whether it can be feasible and possible, the function or feature whose viability has the most uncertainty should be focused on first. If that function or feature is found to be impossible to realize, the artifact as such is impossible. Thus, the wasted effort is minimized.

2. The wasted cost of unnecessary design iteration. In other cases, the justification for this strategy is related to dependencies between functions and features. Regarding the most difficult function, there is usually not much latitude in selecting among different alternatives, but the solution achieved through considerable effort has to be accepted. It may set requirements to the realization of other functions, and if the design of these already has started, rework and iteration may be needed. Thus, by fixing first the most difficult function, the wasted effort in terms of rework and iteration can be minimized.

In passing, it is appropriate to add that the mentioned principle by Peirce seems to be the theoretical justification for using the design structure matrix (DSM) to organize design tasks, seminally proposed by Steward (1981), as well as for the suggestion of rapid prototyping and testing of (partial) solutions, presented in the context of design thinking (Brooks, 2010).

The consequences of this Peirce's principle can be found in how many design methods handle functions. An overall, abstract functional task statement cannot usually be satisfied by an obvious artifact, so it is decomposed in order to identify aspects (subfunctions) with high and low uncertainty associated with them. Peirce tells us that the most economical way of proceeding is by handling the most uncertain aspect first. This principle is not followed by systematic design methods, where all the subfunctions are considered equally important when conducting a morphological search for solution principles. In contrast, it has been in use in the conceptual design method called parameter analysis 
(PA) (Kroll et al., 2001) and its successor, idea-configuration-evaluation (ICE) (Weisbrod and Kroll, 2018), employing a unique design strategy called steepest-first (Kroll et al., 2014): the most challenging aspect of the design task is addressed at any given moment in the process. Based on Peirce, the theoretical justification of this strategy can be pinpointed, and its meaning can be further clarified.

\section{STRATEGIC ABDUCTION WHEN FUNCTIONAL DECOMPOSITION IS DIFFICULT OR IMPOSSIBLE}

Both systematic design and PA/ICE direct the designer to decompose the high-level need statement to functional constituents in a solution-neutral manner. But in many innovative design situations, such a decomposition may not be possible, and can only be carried out after deciding on a solution principle. Consider the following scenario: It is desired to design the means to save the lives of passengers in an airplane that is about to crash due to a malfunction. The overall function may be written as: "bring the passengers to the ground unharmed". How can this function be decomposed in solution-neutral terms? It seems that the only possible course of action is to come up with a solution idea first, and decompose functions later. For example, we might think of deploying parachutes to bring the whole aircraft down slowly, leading to the following top-level decomposition: deploy parachutes + slow descent + touch down. The result will be a conceptual solution as shown in Figure 1.

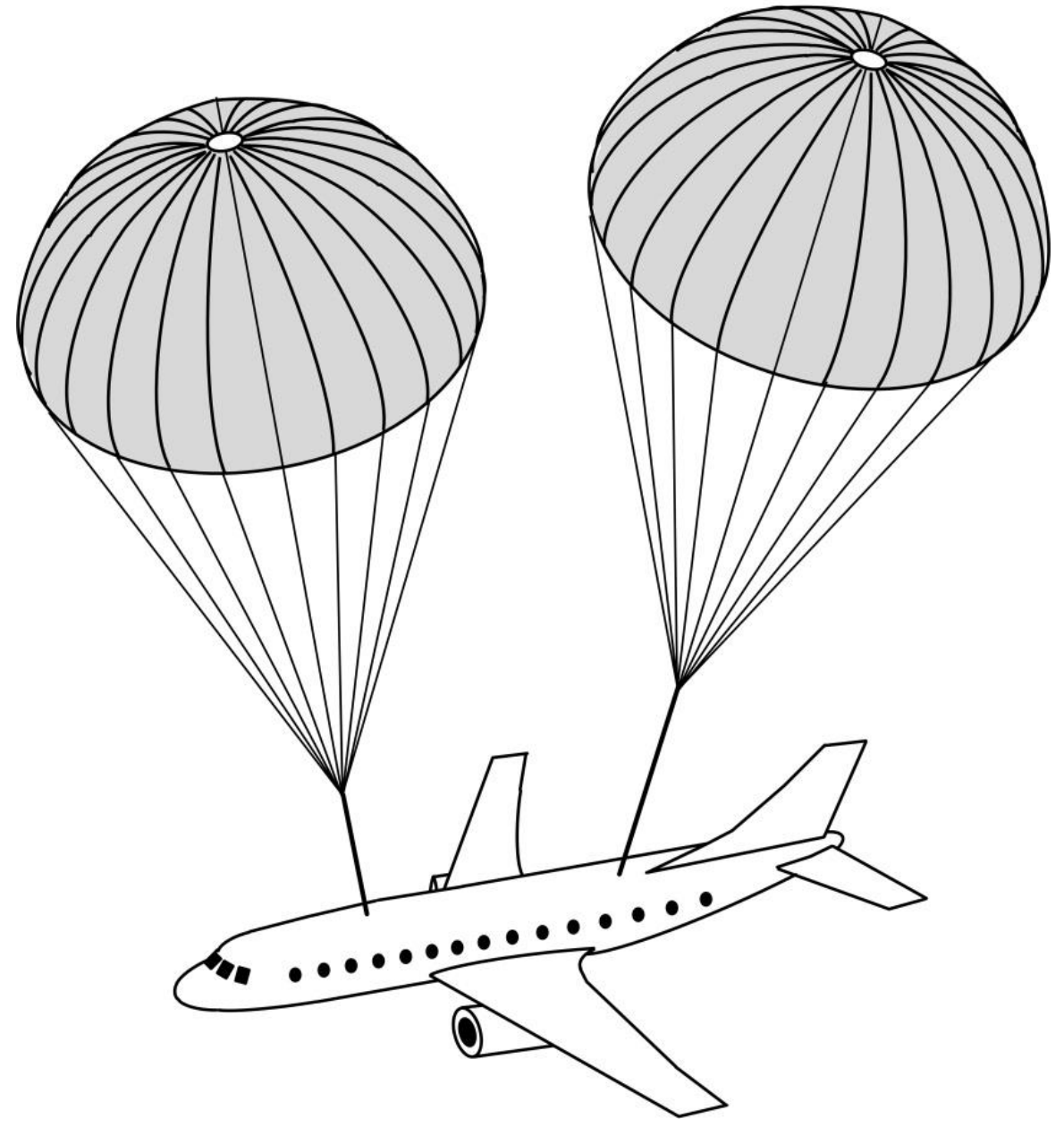

Figure 1. Rescuing passengers by deploying parachutes to bring the airplane slowly to the ground

If, however, the design brief includes the constraint that an engine may be on fire and can explode any second, we may want to get the passengers out of the aircraft quickly, as opposed to prolonging the whole aircraft's descent. In this case the functional decomposition might be: get passengers out of aircraft + deploy parachutes + slow descent + touch down. A further functional decomposition to a more detailed level followed by a morphology, or additional development of the design by another 
method, may lead to solutions based on separating or ejecting the passenger cabin (Figure 2), or providing ejection seats for each passenger.

This example demonstrates that when the design task is very novel, we may be in the anomalous situation of not knowing how to decompose it. To avoid wasting effort on futile attempts, we follow Peirce's principle of reducing the highest uncertainty by changing our strategy to decomposing after "inventing" the main solution idea and not before. This change of strategy can be considered a strategic abduction as it directs the design space exploration in a certain path.

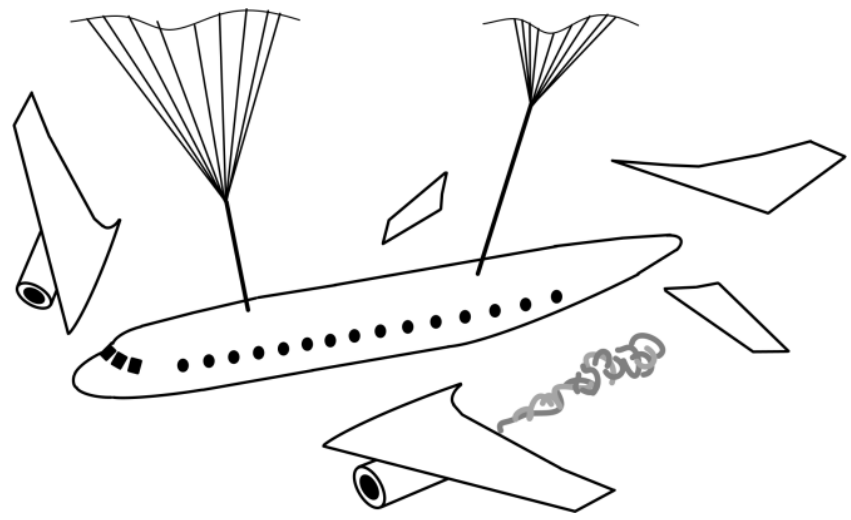

(a)

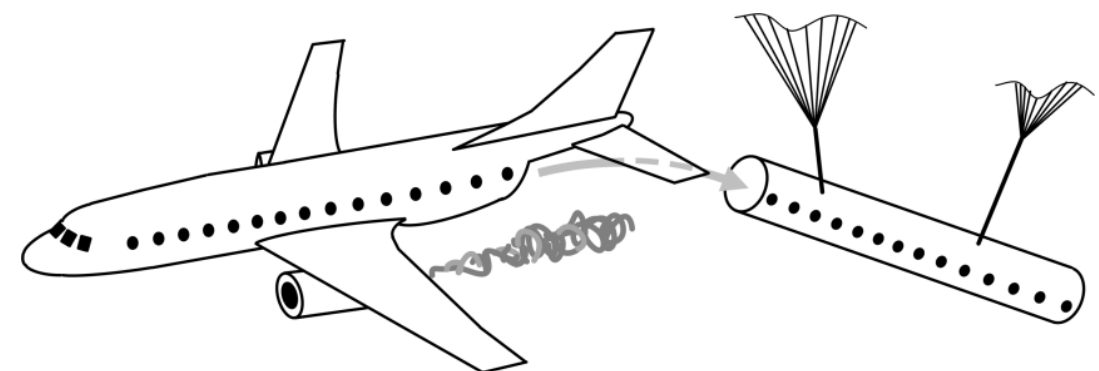

(b)

Figure 2. Rescuing passengers by (a) separating the aircraft appendages from the fuselage, and (b) ejecting the cabin from an opening in the back of the fuselage. In both cases, parachutes are deployed

\section{STRATEGIC ABDUCTION IN DEVELOPING THE FUNCTION STRUCTURE}

The recommended practice when using systematic design methods is to decompose the main function of the designed artifact into smaller subfunctions, and keep decomposing those until reaching subfunctions that cannot be decomposed further or ones that can be satisfied by known devices (e.g., Pahl et al., 2007; Ullman, 2010). The decomposition stage ends with a single function tree or function structure and this is used later as input to the morphology stage where solution principles for each subfunction are listed.

One problem with this decomposition is that the ordering of subfunctions in the function structure can have a significant effect on the resulting design. Consider, for example, the task of designing a handheld nailer as presented in Ulrich and Eppinger (2000). A function diagram as in Figure 3a resulted from decomposing the main function. In searching for solution approaches to the "store or accept energy" subfunction, the designers realized that the nailing operation demanded considerable power, but for only a short duration, so they decided to add an "accumulate energy" subfunction to the top branch of the diagram, Figure 3b. As noted by the authors, the placement of the "accumulate energy" subfunction after the conversion from electrical to mechanical energy will direct the design path to employing springs and moving masses for energy storage. Had this subfunction been placed before the conversion, a very different path - containing accumulation of electrical energy in capacitors - may have resulted. The strategic nature of the abductive decision regarding the ordering of the subfunctions is therefore clear. 


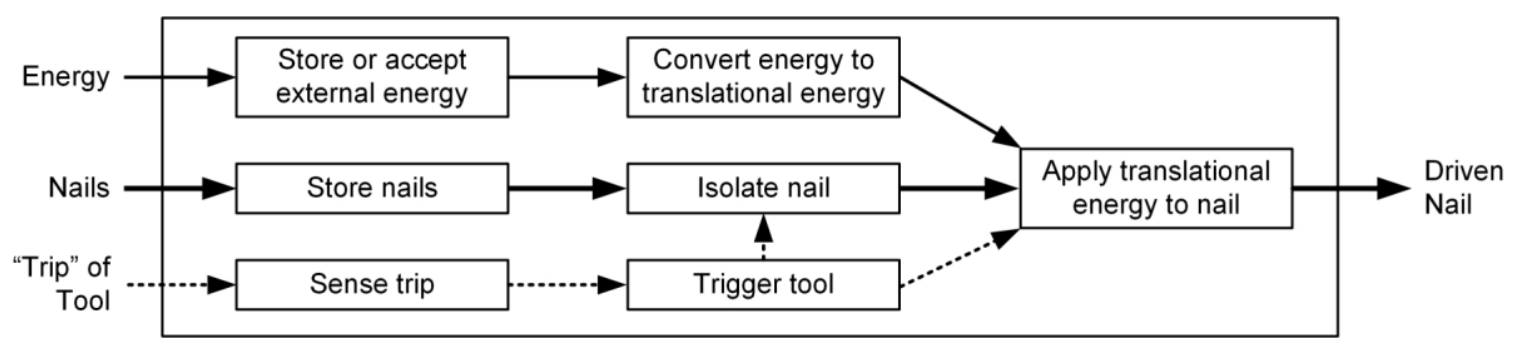

(a)

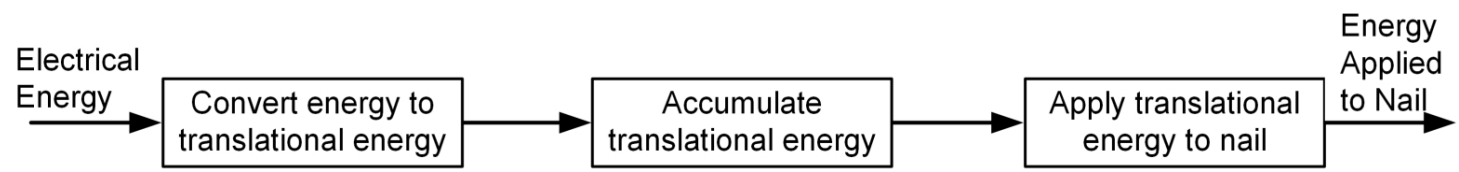

(b)

Figure 3. (a) Function diagram and (b) adding an "accumulate energy" subfunction (redrawn from Ulrich and Eppinger, 2000)

\section{STRATEGIC ABDUCTION IN PRIORITIZING THE DEVELOPMENT ORDER}

After creating a function structure, systematic design methods proceed to list solution principles for each subfunction using the morphology method. No distinction is made at this stage between more and less important functions, and they are all handled concurrently. In contrast, the steepest-first strategy of PA/ICE calls for identifying the most challenging and problematic aspects at any given moment, and focus on them. The following are three examples of the abductive nature of the strategic decisions on the "steepest" subfunction.

\subsection{Autonomous cleaning of windows}

Consider the task of designing an unmanned (robotic) system to clean windows on high rise office buildings to free humans from this dangerous work. The top-level function may be "remove dirt from windows", and suppose the first-level functional decomposition is: "bring a brush/mechanical cleaning device to the window" + "apply water and soap" + "scrub the glass surface" + "collect the dirty water" + "dry the window (optional)". This decomposition already determines a certain solution path (therefore, we may regard it to be strategic), with mechanical action to clean, excluding perhaps other cleaning methods (strong air or water jets, etc.). Now, according to the steepest-first strategy, the most difficult subfunction should be addressed first. But which is the most difficult aspect or subfunction? Many novice designers might start with the cleaning action (rotating brush, water and soap spraying, etc.), as this seems to be at the core of the task. However, further examination would show that there are many known solutions to cleaning, so the biggest challenge may well be getting to the window (think of a robotic device that can move on vertical surfaces or hang from the roof in windy conditions).

\subsection{Mapping ocean currents}

Consider the task of designing a system for mapping the direction and strength of ocean currents. The system should be deployed in large quantities in the oceans and the information gathered would be used to draw current maps for ships and submarines and in marine biology research. Some customer requirements are: (1) Measure flow velocities from $1 \mathrm{~mm} / \mathrm{s}$ to $1 \mathrm{~m} / \mathrm{s}$ in all directions; (2) The maximum depth where measurements are needed is $3000 \mathrm{~m}$; (3) One measurement every hour is required, over a whole year; and (4) The sensors need to stay in the same location for one year.

There seem to be several subfunctions that need to be satisfied: horizontal and vertical deployment of the sensors in the sea, assuring they stay in the same location for a long time, measuring the current velocity and direction and transferring the data. In a class project given to mechanical engineering students, it turned out that the majority of students chose the actual velocity measurement as the most difficult aspect of the problem. However, because there are numerous technologies for measuring fluid velocity, the biggest challenge may well be the placement of each sensor in its location and keeping it there for a long time. So, if the designer imagines a three-dimensional grid of sensors held firmly in place (say, with cables, buoys, anchors, etc.) and focuses on designing the flow meters themselves, 
then this turns out to be a strategic decision that automatically, perhaps inadvertently, eliminates other possibilities, such as fewer sensors that can move between different known locations-for example, a sensor that is lowered and raised on a cable between a buoy and a weight, or a sensor that can "crawl" along such a cable.

\subsection{The Wright Brothers invention of the airplane}

The Wright Brothers realized quite early that three components were needed for an airplane: wings for lift, engine for propulsion, and a control system. The anomalous situation at that point was where to begin? They imagined a mental model (Koskela and Kroll, 2018) of an airplane whose engine fails and the pilot trying to land it safely but loses control and crashes, which is what happened to other aviation pioneers (e.g., Samuel Langley, Figure 4). But if the pilot could maintain control over the incapacitated airplane, he would have landed it safely. Therefore, they concluded, control is more important than propulsion. As for providing lift, they assumed that the knowledge to design the wings was available (from the data generated through many experiments by another contemporaneous aviation pioneer, Otto Lilienthal), so that aspect could be put aside. The success of the Wright Brothers is attributed by Johnson-Laird (2006) to their choice of control as the most important and challenging ("steepest") aspect of the design.

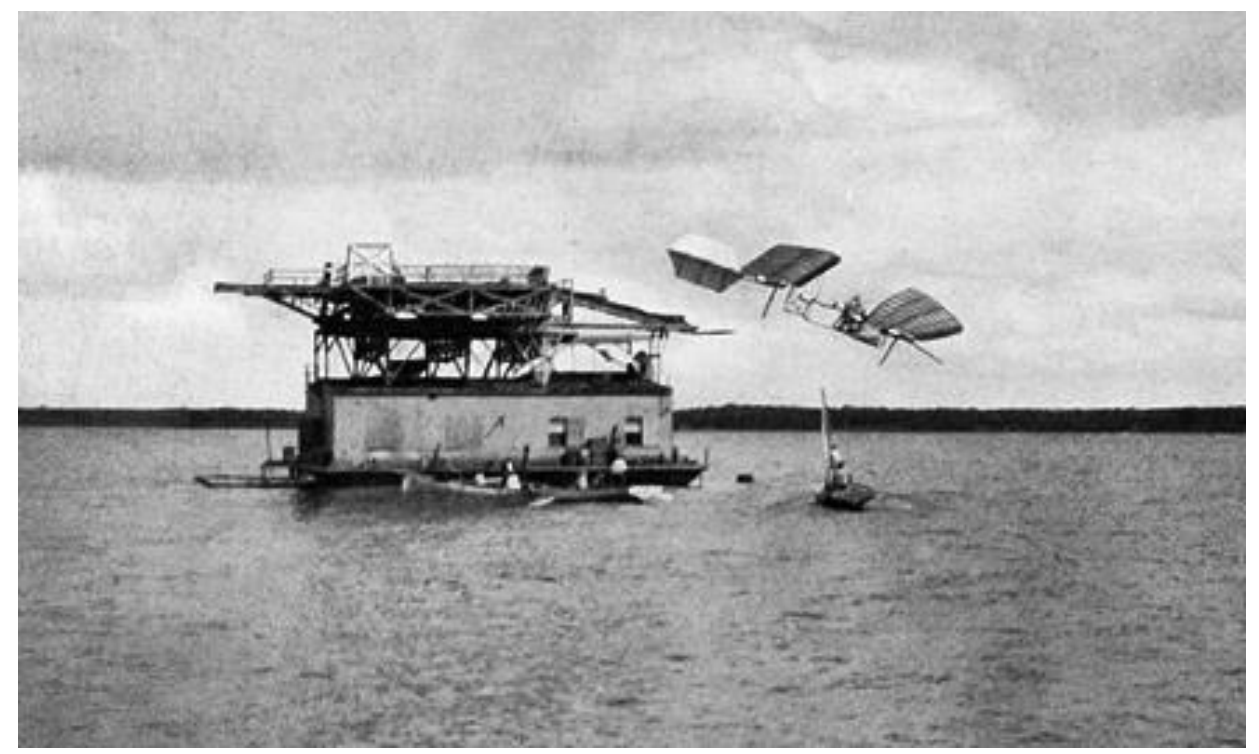

Figure 4. Samuel Langley's Aerodrome plunging into the Potomac River in 1903 (Samuel Pierpont Langley, n.d.)

\section{STRATEGIC ABDUCTION IN MANAGING ITERATIONS}

It seems that the strategy of systematic design does not emphasize the iterative nature of the process. After abstraction of the need and carrying out functional decomposition and morphology, a single or multiple overall solutions emerge, consisting of combinations of the individual solutions for the subfunctions. If one overall solutions turns out to be less favorable, another is generated or selected instead. In contrast, the steepest-first strategy of PA/ICE includes continuous double evaluation of the evolving design (Weisbrod and Kroll, 2018). First, in each design cycle several conceptual solutions are generated for the current "steepest" aspect of the design and one of them is selected for further development based on the designer's evaluation of that concept's chances of success. Second, the developed configuration of the current solution is evaluated for its performance and compliance with the design requirements. Of particular interest is how the overall steepest-first strategy is executed through the choices presented to the designer as a result of the performance evaluation. There are five options, which were implicit in the original PA methodology but became explicit in ICE:

1. If the solution performs satisfactorily, then stop the conceptual design process.

2. If the solution performs well but there is still something missing or an aspect of the solution is still not working well, then carry out another iteration (of finding conceptual solutions to the problematic aspect, selecting among them, and embodying the selected concept as a 
configuration). This option moves the solution forward by staying on the same path.

3. If the solution is flawed, and the problem cannot be fixed easily (as in the previous option), then backtrack to a previously listed conceptual solution that has not been chosen earlier for further development.

4. If the solution is flawed, and the problem cannot be fixed by any of the previously listed conceptual solutions, then a new concept needs to be invented.

5. If the solution is so flawed that even inventing a new concept does not work, the initial formulation of the functional aspects of the design task needs to be examined and perhaps modified.

Option 1 is the stop condition of the process. Option 2 is the desired situation while developing the solution: it keeps the designer on a single path and will be the most economical if successful. Options 3 to 5 represent various degrees of backtracking and re-doing previous choices, and are based on the recognition that design is not only iterative, but also a learning process (Kroll et al., 2014). The knowledge available to the designer keeps changing during design, so early decisions may prove to be less favorable later.

Choosing among the five options at every design cycle is clearly a strategic decision that affects the outcome of the process. It is also abductive, as it begins with an anomalous situation - the need to decide how to proceed - and presents five possible "rules" among which the designer should choose. As common with abductive inferences, the choice of the rule to follow may be based to a large extent on guesses and gut feelings and may lead to a dead end or inferior results. A designer who prefers to take option 2 even when confronted with difficulties may be showing persistence and commitment, or may be suffering from fixation. The strategic decision whether to stay on the current solution path or to switch to another, already existing or totally new path, can be very significant to the final result, but also to the efficiency of the process, as described in Section 2.

\section{DISCUSSION AND CONCLUSION}

The preceding discussions show that strategic abductions, in shaping the design process, count. The prime example, as discussed above, is the invention of the airplane by the Wright brothers, where their success has been attributed to their choice of control as the most important aspect of the design, something that other aviators failed to see. This strategic abduction occurred at the start of the development process. However, as other topics discussed show, abductive inferences are made throughout the process.

What is the upshot of identifying the role of strategic abduction for the design process? Although abduction has been addressed in philosophy of science to a considerable extent, the focus of those studies has not been on understanding the factors hindering or promoting abduction (Paavola, 2014 is a rare exception). However, lead can be taken from other fields where abductions play an important role, especially medicine, where aids for making diagnoses have been developed already for some time.

Somewhat surprisingly, a recent study (Schriger et al., 2017) found that physician judgment is infrequently assessed when the performance of an aid for decision-making is evaluated, and, when reported, the decision aid seldom outperformed physician judgment. The latter finding is similar to the outcome of another study (Sanders et al., 2015): in the limited studies to date, decision aids (termed clinical prediction rules) are rarely superior to clinical judgment.

Although making medical diagnoses of course is different from shaping the design process, the slow progress in aids for medical diagnosis suggests that the human ability to make abductions from incomplete and uncertain data is strong, given experience and expertise. However, such aids, if their capability is near that of an expert, are of course useful in training and for inexperienced professionals. Moreover, a comparison to making medical diagnoses suggests an important difference in the setting. In medical education, the underlying disciplines, such as anatomy, physiology, biochemistry and others, have a strong role. Thus, diseases are not black boxes to medical doctors, but they, more or less, understand the causalities, underlying principles behind the symptoms. Arguably, knowledge of such underlying principles improves abductions. In the case of design processes, such understanding is deficient and unsystematic, as those underlying principles are not usually covered in designer training. The underlying theoretical principles, say of the systematic design method, are not clearly presented in the related literature. On the other hand, the missing attention hitherto to the principle of Peirce about the economy of the research (or design) process suggests that our understanding of the principles is far from complete. As discussed above, Peirce's principle gives a theoretical justification to a number of 
rules (steepest-first, rapid testing cycles, minimizing of unnecessary iteration in general). Thus, exploration and explication of such principles for designer education and for underpinning design models provides one way forward.

Another interesting and somewhat related direction can be captured from the research by Ahmed and Aurisicchio (2007), who observed experienced designers to formulate strategic questions during their design activity, which the designers asked themselves and which were used to progress the design. They found eight such questions, for example: Which are the relevant issues, and which are most important? How much can I expect to achieve if I continue a particular approach? Will an option considered now limit later options in the design process? Which are the limitations of the current design task?

Interestingly, this finding of the role of questions is compatible with the suggestion of Hintikka (2007), to see abductive reasoning as a Socratic process of questions and answers. From a practical viewpoint, the prospect of formulating a set of questions on the strategy of designing, both based on theoretical considerations and empirical findings emerges. Whether such explicit questions, which the designers (or design teams) would ask themselves during the design process, would improve the quality of their abductions, could then be empirically studied. This is another way forward arising from the discussions in this paper.

\section{REFERENCES}

Ahmed, S. and Aurisicchio, M. (2007), "Understanding engineering design activity through questioning", Connected 2007 International Conference on Design Education, pp. 1-7.

Brooks, F. (2010), The design of design: essays from a computer scientist, Addison-Wesley, Upper Saddle River.

Cross, N. (2006), Designerly of ways of knowing, Springer-Verlag, London.

Dorst, K. (2011), "The core of 'design thinking' and its application", Design Studies, Vol. 32, pp. 521-532.

Douven, I. (2017), “Abduction”, The Stanford Encyclopedia of Philosophy (Summer 2017 ed.), Zalta, E.N. (Ed.), from https://plato.stanford.edu/archives/sum2017/entries/abduction/.

Gero, J.S. and Kannengiesser, U. (2004), "The situated function-behaviour-structure framework", Design Studies, Vol. 25, pp. 373-391.

Hatchuel, A. and Weil, B. (2009), "C-K design theory: an advanced formulation", Research in Engineering Design, Vol. 19, pp. 181-192.

Hintikka, J. (2007), Socratic epistemology: explorations of knowledge-seeking by questioning, Cambridge University Press, Cambridge.

Johnson-Laird, P.N. (2006), How we reason, Oxford University Press, New York.

Koskela, L. and Kroll, E. (2018), "Model-based abduction in design”, Design Computing and Cognition '18, Lecco, June 30-July 1, Springer, Cham, pp. 279-298.

Koskela, L., Paavola, S. and Kroll, E. (2018), "The role of abduction in production of new ideas in design", In: Vermaas, P.E. and Vial, S. (Eds.), Advancements in the Philosophy of Design, Springer, Cham. Ch. 8.

Kroll, E. (2013), "Design theory and conceptual design: contrasting functional decomposition and morphology with parameter analysis", Research in Engineering Design, Vol. 24, pp. 165-183.

Kroll, E., Koskela, L. (2016), "Explicating concepts in reasoning from function to form by two-step innovative abductions", Artificial Intelligence for Engineering Design, Analysis and Manufacturing (AI EDAM), Vol. 30, pp. $125-137$.

Kroll, E., Condoor, S. and Jansson, D. (2001), Innovative conceptual design: theory and application of parameter analysis, Cambridge University Press, Cambridge.

Kroll, E., Le Masson, P. and Weil, B. (2014), "Steepest-first exploration with learning-based path evaluation: uncovering the design strategy of parameter analysis with C-K theory", Research in Engineering Design, Vol. 25, pp. 351-373.

Maher, M.L. (2001), “A model of co-evolutionary design”, Engineering with Computers, Vol. 16, pp. 195-208.

March, L. (1976), "The logic of design and the question of value", In: March, L. (Ed.), The Architecture of Form, Cambridge University Press, Cambridge, pp. 1-40.

Paavola, S. (2014), "From steps and phases to dynamically evolving abduction", presented at The Charles $S$. Peirce International Centennial Congress 2014: Invigorating Philosophy for the 21st Century, July 16-19, Lowell.

Pahl, G., Beitz, W., Feldhusen, J. and Grote, K.H. (2007), Engineering design: a systematic approach, 3rd ed. Springer-Verlag, London.

Peirce, C.S. (1967), "Note on the theory of the economy of research", Operations Research, Vol. 15, No. 4, pp. 643-648.

Roozenburg, N.F.M. (1993), “On the pattern of reasoning in innovative design”, Design Studies, Vol. 14, pp. 4-18. 
Samuel Pierpont Langley (n.d.), In Wikipedia. Retrieved December 15, 2018, from https://en.wikipedia.org/w/index.php?title=Samuel_Pierpont_Langley\&oldid=872269153.

Sanders, S., Doust, J. and Glasziou, P. (2015), “A systematic review of studies comparing diagnostic clinical prediction rules with clinical judgment", PLOS one, Vol. 10, p. e0128233.

Schriger, D.L., Elder, J.W. and Cooper, R.J. (2017), "Structured clinical decision aids are seldom compared with subjective physician judgment, and are seldom superior”, Annals of Emergency Medicine, Vol. 70, pp. 338-344.

Steward, D.V. (1981), "The design structure system: a method for managing the design of complex systems", IEEE transactions on Engineering Management, Vol. 3, pp. 71-74.

Suh, N.P. (1990), The principles of design, Oxford University Press, New York.

Ullman, D.G. (2010), The mechanical design process, 4th ed., McGraw-Hill, New York.

Ulrich, K.T. and Eppinger, S.D. (2000), Product design and development, 2nd ed., McGraw-Hill, New York.

Weisbrod, G. and Kroll, E. (2018), "Idea-configuration-evaluation (ICE): development and demonstration of a new prescriptive model of the conceptual engineering design process based on parameter analysis and $\mathrm{C}-\mathrm{K}$ theory", Research in Engineering Design, Vol. 29, pp. 203-225. 